\title{
PRESCRIBING PATTERNS OF NON-STEROIDAL ANTI-INFLAMMATORY DRUGS IN OUTPATIENT CLINICS AT ROYAL REHABILITATION CENTER IN KING HUSSEIN MEDICAL CENTER
}

Kholoud Z. Qoul", Ikbal N. Thuheerat, Nebal Abu Ashor, Neris M. Hakuz.

King Hussein Medical Center, Royal Medical Services, Jordan Armed Forces

\section{ABSTRACT}

Objective: The purpose of this paper is to analyze the prescribing patterns for both conventional and non-conventional non-steroidal anti-inflammatory drugs (NSAIDs) with concomitant gastro-protective agents, at the outpatient clinic at Royal Rehabilitation Center (RRC) in King Hussein Medical Center (KHMC) in Jordan.

Methods: A retrospective study was conducted at the outpatient clinic pharmacy in RRC. A total of 25,692 prescriptions were reviewed. Collected data includes: percentage of each type of NSAIDs, dosage form, percentage share for each selective and non-selective NSAID, and concomitant therapy with gastroprotective agent.

Results: $52 \%$ of the collected prescriptions contain NSAIDs. $76 \%$ of the prescriptions are for women and $24 \%$ are for men. Age of patients included in the prescription ranges between 16 and 80 years, with a mean of $59.3 \pm 15.8$ years. Indications for NSAIDs are $58.3 \%$ for osteoarthritis, $12.1 \%$ for rheumatoid arthritis, and $20.1 \%$ for orthopedics pain. Additionally, $96.4 \%$ of prescriptions are for conventional NSAIDs, while only $3.6 \%$ prescriptions are for the selective COX-2 inhibitors. Furthermore, diclofenac topped the list with $83.74 \%$ of prescriptions of NSAIDs. Concomitant therapy with gastroprotective agents was reported in $71.2 \%$ of prescriptions. Famotidine is the most prescribed gastroprotective agents followed by antacid and omprazole.

Conclusions: In summary, Diclofenac was the most prescribed NSAIDs as a result of its low price and availability in different dosage forms. Conventional NSAIDs combined with a gastroprotective agent is the most appropriate first-line NSAIDs therapy for many patients. To minimize the occurrence of gastrointestinal toxicity, the study suggests adopting the National Institute of Clinical Excellence (NICE) guidance or the American College of Gastroenterology recommendations. KEYWORDS: conventional NSAIDs, non-conventional (selective COX-2 inhibitors) Non-steroidal anti-inflammatory drugs; gastro-protective agents,

\section{INTRODUCTION}

No on-steroidal

anti-inflammatory

drugs groups of pharmaceutical agents. Worldwide, over 30 million people use NSAIDs every day $(1,2)$. In the USA alone, the annual number of prescriptions exceeds 111 million. Additionally, NSAIDs account for more than $60 \%$ of over-the-counter (OTC) analgesic market (3).Unfortunately, Jordan and other Arab countries lack any published official information in relation to the volume of NSAIDs use (4).

NSAIDs are utilized widely for a variety of disorders, which are related to pain and inflammation including musculoskeletal disorders (5). However, clinical and epidemiological studies have shown that the use of NSAIDs causes adverse reactions in the gastrointestinal system. In particular, NSAIDs have both bleeding and dyspepsia effects $(6,7)$. These adverse effects are a consequence of NSAIDs ability to inhibit cyclooxygenase-1 (COX-1) in the gastrointestinal tract (8). The selective COX-2 inhibitors emerged as potentially gastro-friendly NSAIDs $(9,10)$. Recent studies revealed cardiovascular adverse effects with the use of COX-2 selective inhibitors, which raised concerns among both prescribers and consumers (11).

The British National Formulary (BNF) lists more than 20 NSAIDs that are available in at least 40 different formulations. Patients' response to NSAIDs is highly variable (12). There are several factors that contribute to patients' preference for one drug over another such as treatment efficacy, time and chance to benefit, potential side effects, ease of administration, cost, physician and patient beliefs drug interaction, severity of disease, and health status $(13,14,15)$.

The purpose of this study is to analyze the prescribing patterns of conventional NSAIDs, nonconventional (selective COX-2 inhibitors) and concomitant gastro-protective agents. Data for this research project were collected from the out-patient pharmacy of the Royal Rehabilitation Center (RRC) at King Hussein Medical Center (KHMC) in Jordan. The results of the study provide valuable information to scholars, practitioners, and policy makers.

\section{METHODS}

A retrospective study was conducted at the outpatient pharmacy in RRC during the last quarter of 2012. The study was approved by the Ethical 
Committee at the Royal Medical Services (RMS). For the period of the study, the researchers reviewed all prescriptions, which totaled 25,692 prescriptions. Prescriptions surveys are regarded as effective means to provide insights into the underlying prescription patterns.

The collected data includes information as follows: age, sex, indication for use, average number of drugs per prescription, percentage of each type of selective and non-selective NSAIDs, dosage form, and concomitant therapy with gastroprotective agents. Prior to conducting the study, the researchers' obtained the approval of the Ethical Committee at the Royal Medical Services (RMS).

\section{RESULTS AND ANALYSIS}

The total number of prescriptions in RRC during the period of study totaled 25,692 prescriptions. $52 \%$ of these prescriptions include NSAIDs. Out of the total number of NSAIDs prescriptions, $76 \%$ are for women, while the remaining $24 \%$ are for men. Age of patients ranges between 16 and 80 years, with a mean of $59.3 \pm 15.8$ years. The average number of drugs per prescription is 3.76 with a range of $1-8$ drugs. About $72 \%$ of the patients use 3 or more medications. Table 1 shows descriptive statistics for the collected data in relation to age and indication for use. As shown in Table 1, 58.3\% of NSAIDs are used for osteoarthritis, $20.1 \%$ for orthopeadic pain, $12.1 \%$ for rheumatoid arthritis, and $9.5 \%$ for other musckeloskeletal disorders.

Table 1: Descriptive statistics for age and indication for use

\begin{tabular}{ll}
\hline Age (years) & Percentage \\
\hline $16-39$ & 19.4 \\
$40-59$ & 40.0 \\
$60-80$ & 40.6 \\
\hline Indication for use & \\
\hline Osteoarthritis & 58.3 \\
Orthopedic pain & 20.1 \\
Rheumatoid Arthritis & 12.1 \\
Other musckeloskeletal disorders & 9.5 \\
\hline
\end{tabular}

Figure 1 shows the percentage for each NSAID. $96.42 \%$ prescriptions are for conventional NSAIDs, while only $3.58 \%$ prescriptions are for the newer selective COX-2 inhibitors. The majority of NSAIDs is prescribed in oral dosage form (73.42 $\%)$ followed by topical (18.24\%) and rectal (7.33 $\%)$. The injectable dosage form is the least prescribed with only (1\%) of all prescribed NSAIDs.
Figure 1 reveals that Diclofenac tops the list and is the most frequently prescribed NSAIDs with $83.74 \%$. Dosage forms include tablet $(58.72 \%)$, gel (18.24\%), and suppository (5.78\%). Ibuprofen tablets and Indomethacin capsule are prescribed $5.63 \%$ and $3.84 \%$ respectively. COX-2 inhibitor that is available in RRC is meloxicam in both tablet and suppository dosage forms. These two forms were prescribed $2.85 \%$ and $0.75 \%$ respectively. 




Figure 1: Average prescription rates for individual NSAIDs

Concomitant therapy with gastoprotective agents were reported in $71.16 \%$ of prescriptions. Figure 2 shows gastroprotective agents prescribed. Famotidine is the most commonly prescribed one $(86.30 \%)$, followed by antacid (10.85\%) and omprazole $(2.85 \%)$.

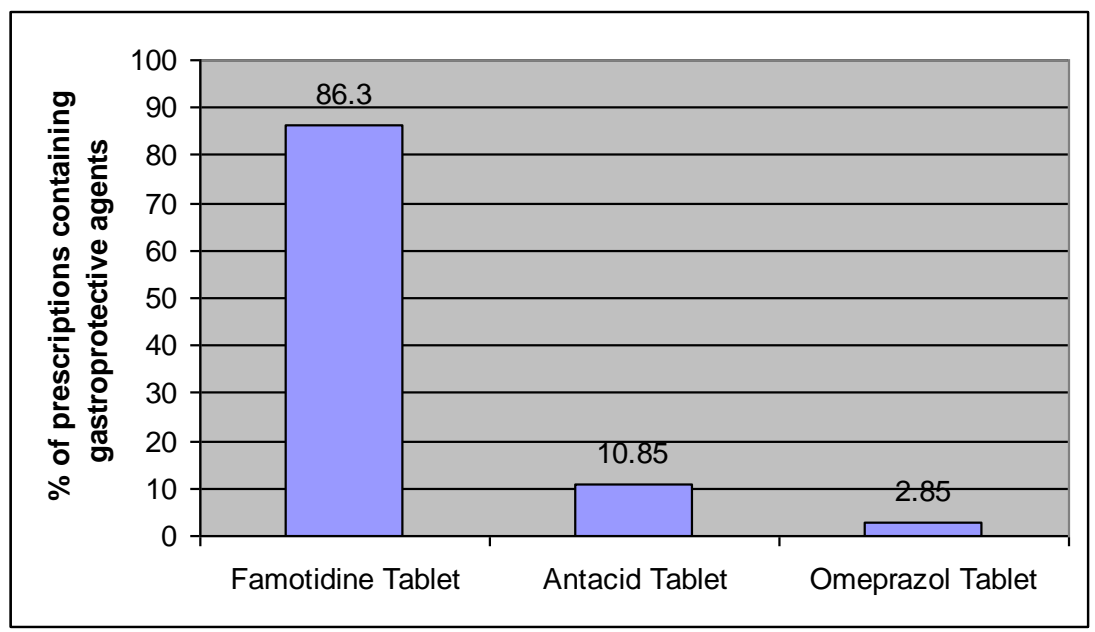

Figure 2: Gastroprotective agents prescribed

\section{DISCUSSION}

The result from this study shows that the use of selective COX-2 at RRC was limited to a mere $3.58 \%$ of prescriptions. These results are consistent with Zandman and Langville study, which reported that only $4 \%$ of patients were treated with COX-2 inhibitors (16). To the contrary, Thompson et al. study indicated that $24 \%$ of prescriptions are COX-2 inhibitors (17).

The conventional and the selective NSAIDs are broadly prescribed for indications including osteoarthritis, rheumatoid arthritis, and acute pain. Both drugs have identical clinical efficacy $(18,19$, 20). COX-2 inhibitors are more costly compared to nonselective NSAIDS, which results in lower COX2 prescriptions compared to nonselective NSAIDs prescriptions $(21,22)$.

Even though that COX-2 selective inhibitors were developed to achieve safer renal and gastrointestinal profile compared to non-selective NSAIDs, but these former drugs have a pattern of nephrotoxicity and drug interactions similar to those of conventional NSAIDs $(23,24)$. Rostom et al. conducted a Cochrane meta-analysis that compared gastrointestinal safety of coxibs and NSAIDs (25). The study advocated that coxibs are significantly associated with fewer gastroduodenal ulcers and ulcer complications. Yet, a previous study showed 
no evidence that coxibs are less toxic to the gastrointestinal tract than conventional NSAIDs combined with a gastro protective agent such as a proton pump inhibitor (PPI), especially in patients with high risk of developing gastrointestinal adverse events $(26,27)$.

Additionally, clinical, experimental, and reviews suggest that the use of selective COX-2 inhibitors is associated with the increase in systolic blood pressure and cardiovascular morbidity due to myocardial infarction (MI) and cardio vascular system (CVS) toxicity (28, 29, 30, 31,32,33 ). Convincingly, studies by Lamarque and Bannwarth support reversing the trend back to the use of conventional NSAIDs $(34,35)$. More recently, Olsen et al.indicated that both selective and conventional NSAIDs have potential cardiovascular risk. The authors argued that both drugs appear to increase the risk of subsequent cardiac events following MI (36).

Table 1 shows that NSAIDs use was significantly higher in female (76\%) compared with male (24\%). The dominant indication for prescription of NSAIDs is osteoarthritis with $(58.3 \%)$ of prescriptions. This is followed by orthopeadic pain $(20.1 \%)$, rheumatoid arthritis (12.1 $\%)$, and musckeloskeletal disorders $(9.5 \%)$. These results are consistent with the argument of Bannwarth, who concluded that these conventional NSAIDs combined with a gastroprotective agent would be the most appropriate first-line NSAID therapy in many patients with osteoarthritis (37) . Kean suggested re-establishing the conventional NSAIDs as the preferred choice in the management of arthritis and musculoskeletal disorder (38).

Figure 1 shows that $(83.74 \%)$ of the prescriptions were for diclofenac, followed by (5.63\%) for ibuprofen. These results are similar to Fosb $\varnothing 1$ et al. study, which investigated the pattern of use of non-steroidal anti-inflammatory drugs (NSAIDs). Fosbøl et al. concluded that Ibuprofen and diclofenac were the most frequently used conventional NSAIDs (39). While IMS Health reports that diclofenac is more common prescribed in the UK. However, in the USA, ibuprofen and naxproxen are the most commonly prescribed NSAIDs (40). Another study by Al-bsoul et al. showed that the diclofenac is the preferred NSAID among the Jordanian patients. According to the authors, this preference is attributed to availability in different dosage forms and low price (4).

The use of Diclofenac is distributed as follows: tablet $(58.72 \%)$, gel $(18.24 \%)$, suppository $(5.78 \%)$, and injection (1\%). The increased utilization of gel is due to the fact that this topical compound offers the advantage of relieving symptoms of osteoarthritis with lower incidence of systemic adverse effect (20). Injectable dosage form was the least prescribed with only (1\%). According to Drug and Therapeutic Bulletin the use of injection is seldom preferred rout for musckeloskeletal disorders(41).

This study showed that there is a high prescription rate of gastro protective agent concomitantly with NSAIDs. Among the total (8720) prescriptions of conventional systemic NSAIDs, (71.16\%) are co-prescribed with gastroprotective agents. Figure 2 shows that Histamine type 2 receptor antagonist $\left(\mathrm{H}_{2} \mathrm{RA}\right)$, available at RRC as Famotidine $40 \mathrm{mg}$ tablet, was prescribed in $(86.30 \%)$ of prescriptions, representing the most frequent gastroprotective agent prescribed. This is followed by antacids and proton pump inhibitor ( PPI), available at RRC as omprazole, that represented $(10.85 \%)$ and $(2.85 \%)$ of prescriptions respectively. These results are consistent with recent recommendations by Lanza et al. and Brown et al. which suggested that the use of co-therapy with misoprostol, histamine type-2 receptor antagonists $\left(\mathrm{H}_{2} \mathrm{RAs}\right)$, or PPIs with nonsteroidal anti-inflammatory and/or the use of cyclooxygenase- 2 selective inhibitors decrease gastrointestinal toxicity $(42,37)$.

In RRC, H2RA is prescribed in standard dose (famotidine $40 \mathrm{mg}$ ). Rostom et al. ( conducted Cochrane review and Koch et al. conducted metaanalysis. They found that standard doses $(40 \mathrm{mg}$ tablet) of $\mathrm{H}_{2} \mathrm{RAs}$ were effective at reducing the risk of duodenal but not gastric NSAID associated ulcers $(43,44)$. Rostom et al. concluded that high dose $\mathrm{H}_{2} \mathrm{RAs}$ is effective at preventing chronic NSAIDs related duodenal and gastric ulcer (43).

A study that was conducted in Italy found that $35 \%$ of the chronic NSAIDs users, who reported gastrointestinal symptoms, prescribed a drug for acid-related disorders, while only $14 \%$ used daily a PPI (45). Additionally, another study from France found that the proportion of prescriptions combining NSAIDs and gastroprotective agents was $29.5 \%$, with omprazole accounted for $58 \%$ of the co-prescriptions and misoprostol for $29.5 \%$ (5). Furthermore, Lazzaroni and Porro indicated that PPI are efficient in the treatment as well as prophylaxis 
of non-steroidal anti-inflammatory drug related mucosal lesions of gastrointestinal tract (46).

Figure 2 shows that $(10.85 \%)$ of the prescriptions are NSAIDs-antacid combination, which is much lower than the percentage $(87.3 \%)$ reported by Liu et al. (47).

The sample of this study consists of $(40.6 \%)$ of elderly patients. The risk of NSAIDrelated gastrointestinal toxicity is increased in this group of patients by more than 2 folds (48). As a result, this group of patients should be prescribed appropriate gastroprotective agents and/or $\mathrm{COX}-2$ inhibitor.

\section{CONCLUSION AND RECOMMENDATION}

The study concludes that the traditional NSAIDs combined with a gastroprotective agents are the most appropriate first-line NSAID therapy for many patients. To minimize the occurrence of gastrointestinal toxicity, it is advised to use the National Institute of Clinical Excellence (NICE) guidance or the American College of Gastroenterology recommendations.

Future research should incorporate patient related factors such as the concurrent low dose aspirin use, prior upper GI events, concomitant use of anticoagulation, or corticosteroid therapy and use of multiple NSAIDs or high dose NSAIDs.

\section{REFERENCES}

1. Singh, G., and Traidafilopulos, G.: Epidemiology of NSAIDs induced gastrointestinal complications. Journal of Rheumatology 1999; 26: 18-24.

2. Singh, G.: Gastrointestinal complications of prescription and over- the -counter nonsteroidal anti-inflammatory drugs: a view from the ARAMIS data base. Arthritis, Rheumatism, and Aging Medical Information System. Am J Ther.; 2000 ; 7:115-121.

3. Laine, L.: Approaches to nonsteroidal antiinflammatory drug use in the high-risk patient. Gastroenterology; 2001; 120:594-606.

4. Albsoul, A.; Jabateh, S.;Abedl-Hafiz, S;Safi, S.: Awareness and frequency of potential side effects on non-steroidal anti-inflammatory drugs among the Jordanian patient population. Saudi Medical Journal ; 2004;25 (7): 907-911.

5. Clinard, F.; Bardon, M.;Sgro, C.;Lefevre, N. ;Raphael, F. ;Paille, F.;Dumas, M. ;Hillon, P. and Bonithon-Kopp, C.: Nonsteroidal anti-inflammatory and cytoprotective drug co-prescription in general practice . A general practitioner-based survey in France. European Journal of Clinical Pharmacology; 2001; 57 (10): 737-743.

6. Rodriguez, G.; and Jick H. : Risk of upper gastrointestinal bleeding and perforation associated with individual non-steroidal anti-inflammatory drugs. Lancet :1994;343: 796-773.

7. Langman, M.;Weil, J.; and Wainwright, P.: Risks of bleeding peptic ulcer associated with individual non-steroidal anti-inflammatory drugs Lancet: 1994; 343:1075-1078.

8. Sharma, T.; Dutta, S. ;and Dhasmana, D.:Prescribing pattern of NSAIDs in orthopaedic OPD of a tertiary care teaching hospital in Uttaranchal, JK Sceince; 2006;8 (3): 160-162.

9. Graumlich, J.: Preventing gastrointestinal complications of NSAIDs: risk factors, recent advances, and latest strategies. Post Grad Medical ; 2001; 109: 117-128.

10. Silverstein, F.; Faich, G.; Goldstein, J.; Simon, L.; Pincus, T.; Whelton, A., et al.: Gastrointestinal toxicity with Celecoxib versus non-steroidal antiinflammatory drugs for osteoarthritis and rheumatoid arthritis. Jama Express; 2000; 284:1247-1255.

11. Elsy, M.; Ajitha, K.; Sanalkumar, K.; Joythish, K.; and Kuttichira, P.: Prescribing pattern of analgesics in orthopaedic department of an Indian tertiary care teaching hospital in Kerala. Kerala Medical Journal; $2011 ; 4: 149-152$.

12. Walker, JS; Seather-Reid, RB and Carmody, JJ.: Nonsteroidal anti-inflammatory drug in rheumatoid arthritis and osteoarthritis: support for the concept of " responders" and " non-responders". Arthritis Rheum; 1997; 40: 1044-1954.

13. Wegman, A.; Van der Windt, D.; Van Tulder, M. et al.: Non-steroidal anti-inflammatory drugs or acetaminophen for osteoarthritis of the hip or knee? A systemic review of evidence and guidelines. The Journal of Rheumatology ; 2004;31:344-354.

14. Pincus T.; Koch G.; Sokka T.; et al.: A randomized, double - blind, crossover clinical trial of diclofenac plus misoprostol versus acetaminophen in patients with osteoarthritis of the hip or Knee. Arthritis and Rheumatism; 2001 ; 44:1587-1598.

15. Franenkel L.; Bogardus Jr.; St., Concato, J.; and Wittink, D.: Treatment options in knee osteoarthritis .The patient's perspective. Archives of Internal Medicine ; 2004;164:1299-1304.

16. Zandman-Goddard G.; and Langville, P.: The lack of awareness of the Israeli population regarding gastrointestinal complications from nonsteroidal anti-inflammatory drugs. Harefuah ; 2001;140 (6): 476-478.

17. Thompson, P.; Tee, L.; Bird, M.; Quincey, D.; and Liddiard, G.:Long-term NSAID use in primary care: changes over a decade and NICE risk factors for gastrointestinal adverse events. Rheumatology; 2005; 44:1308-1310.

18. Day, R.;:Morrison, B.; Luza, A.; et al. : A randomized trial of the efficacy and tolerability of the COX-2 inhibitor rofecoxib vs ibuprofen in 
patients with osteoarthritis. Arch Intern Med; 2000; 160: 1781-1787.

19. Saag, K.; Van der Heijde, D.; Fisher, C.; et al.: Rofecoxib, a new cyclooxygenase 2 inhibitor, shows sustained efficacy, comparable with other nonsteroidal anti-inflammatory drugs: a 6-week and a 1-year trial in patients with osteoarthritis. Arch Fam Med; 2000; 9, 1124-1134.

20. Heyneman, C.; Lawless-Liday, C.; and Wall, G.: Oral versus topical NSAIDs in rheumatic diseases. A comparison. Drugs; 2000; 60: 555-574.

21. Jose, V.; and Antonyn, T.: Recent trends in the utilization of NSAIDs in a tertiary care Hospital. Indian Journal of Pharmacology; 2003; 35: 318.

22. Tiwari, H.; Kumar, A.; Kulkarni, S.: Prescription monitoring of antihypertensive drug utilisation at the Panjab University Health Centre in India. Singapore Medical Journal; 2004; 45: 117-120.

23. Perazella, M.: COX-2 selective inhibitors: analysis of the renal effect. Expert Opinion on Drug Safety; 2002; 1:53-64.

24. Mahajan, A. and Sharma, A.:COX-2 selective nonsteroidal anti-inflammatory drugs: current status. The Journal of the Association of Physician of India; $2005 ; 53: 200-204$.

25. Rostom, A.;Muir, K.; Dube, C.; Jolicoeur, E.; Boucher, M.; Joyce, J.; Tugwell, P.;Wells, G.:Gastrointestinal safety of cyclooxygenase-2 inhibitors: a Cochrane collaboration systematic review. Clin Gastroenterol Hepatol; 2007;5: 818828.

26. Chan, F.;Hung, L.; Suen, B. et al.: Celecoxib versus diclofenac and omprazole in reducing the risk of recurrent ulcer bleeding in patient with arthritis. The New England Journal of Medicine; 2002; 347: 2104-2110.

27. Spiegel, B.; Chiou, C.; and Ofman, J.:Minimizing complications from nonsteroidal anti-inflammatory drugs:cost-effectiveness of competing strategies in varying risk groups. Arthritis and Rheumatism; 2005; 53: 185-197.

28. Furberg, C.; Psaty, B.; and FitzGeral, G.: Parecoxib, Valdecoxib, and cardiovascular risk. Circulation; 2005; 111: 249.

29. Graham, D. ; Campen, D.; Hui, R.; Spence, M. ; Cheetam, C. ;Levy, G. ,Shoor, S.; and Ray, W. : Risk of acute myocardial infarction and sudden cardiac death in patients treated with cyclooxygenase-selective and non-selective nonsteroidal anti-inflammatory drugs :nested case-control study. Lancet; 2005; 365: 475-481.

30. Johnsen, S.; Larsson, H.; Taone, R., et al. : Risk of hospitalization for myocardial infarction among users of rofecoxib, celecoxib, and another NSAIDs. Archives of Internal Medicine ;2005 ; 165: 978984.

31. Andersohn, F.; Suissa, S.; Garbe, E. :Use of firstand second-generation cyclooxygenase-2-selective
Nonsteroidal anti-inflammatory drugs and risk of acute myocardial infarction. Circulation. ; 2006 ; 113:1950-1957.

32. Tandon, V.: VR pain killers and cardiovascular toxicity. Health Line Family Medical Journal; 2006; 4: 33-34.

33. Trelle, S.; Reichenbach, S.; Wandel, S.; Hildebrand, P.; Tschannen, B., Villiger; P. Egger M.; Juni, P.: Cardiovascular safety of non-steroidal antiinflammatory drugs: network meta-analysis. BMJ; 2011; 342: c7086.

34. Lamarque D.: Safety of selective inhibitors of inducible cyclooxygenase - 2 taken for a long period. Bull Cancer; 2004; 91: 117-124.

35. Bannwarth B.: Do selective cyclo-oxygenase-2 inhibitors have a future? Drug Safety; 2005; 28(3):183-189.

36. Olsen,A.; Fosbøl, E.; Lindhardsen,J., et al.: Longterm cardiovascular risk of nonsteroidal antiinflammatory drug use according to time passed after first-time myocardial infarction: a nationwide cohort study. Circulation; 2012; 126(16): 1955-63.

37. Brown, T.; Hooper, L.; Elliott, R.; Payne, K.; Webb, R.; Roberts, C.; Rostom A.; and Symmons, D.: A comparison of the cost-effectiveness of five strategies for the prevention of non-steroidal antiinflammatory drug-induced gastrointestinal toxicity: a systematic review with economic modeling. Health Technol Assess; 2006; 10: 1-183.

38. Kean, W.:The use of NSAIDs in rheumatic disorders: a global perspective. Japanese Society of Pharmaceutical Health Care and Sciences; 2005; 30 (3):224-230.

39. Fosbøl, E. ;Gislason, G.; Jacobsen, S.; Abildstrom, S.; Hansen, M., Schramm, T.; Folke, F.; Sørensen, R.; Rasmussen, J.; Køber, L.; Madsen, M.; TorpPedersen, C.,. The pattern of use of non-steroidal anti-inflammatory drugs (NSAIDs) from 1997 to 2005: a nationwide study on 4.6 million people. Pharmacoepidemiol Drug Safety; 2008; 17(8): 822833.

40. IMS health IMS MIDAS quantum based on selected markets (ATC=M1C oral solid forms only. (2008) US\$ Actual growth in US\$ CER.)Q4

41. Drug and Therapeutic Bulletin: Rational use of NSAIDs for musculoskeletal disorder. ; 1994; 32:91-95.

42. Lanza, F.; Chan, F.; and Quigley, E.: Practice Parameters Committee of the American College of Gastroenterology: Guidelines for prevention of NSAID related ulcer complications. American Journal Gastroenterology; 2009; 104:728-738.

43. Rostom, A.; Dube, C.; Wells, G.; Tugwell, P.; Welch, V.; Jolicoeur, E.; and McGowan, J.: Prevention of NSAID-induced gastroduodenal ulcers. Cochrane Database Syst ; 2002; 4: 2296.

44. Koch, M.; Dezi, A.; Ferrario, F.; and Capurso, I. :. Prevention of nonsteroidal anti-inflammatory drug- 
induced gastrointestinal mucosal injury. A metaanalysis of randomized controlled clinical trials. Arch Intern Med; 1996; 156: 2321-2332.

45. Silvani, M.; Motola, D.; Poluzzi,E.; Bottoni, A.; De Ponti, F.; Vaccheri, A.; and Montanaro, N. : Gastro-intestinal problems and concomitant medication in NSAID users: additional findings from a questionnaire-based survey in Italy. European Journal of Clinical Pharmacy; 2006; 62 (3): 235-241.

46. Lazzaroni, M. and Porro, G.: Prophylaxis and treatment of non-steroidal anti-inflammatory drug- induced upper gastrointestinal side-effects. Digestive and Liver Disease; 2001; 33 (2):S44-S58.

47. Liu, J.; Chen, T.; and Hwang, S.,: Concomitant prescription of non-steroidal anti-inflammatory drugs and antacids in the out-patient setting of a medical center in Taiwan: a prescription database study. European Journal of Clinical Pharmacology; 2001; 57 (6-7): 505-508.

48. Bradou,M. and Barkun,A.: Preventing the gastrointestinal adverse effects of nonsteroidal antiinflammatory drugs: From risk factor identification to risk factor intervention. Joint Bone Spine; 2010; 77: 6-12. 